\title{
Pacemaker Lead Associated Superior Vena Cava Syndrome Treated By Veno-Atrial Bypass
}

\author{
Thilina Gunawardena, MBBS, MD, MRCS*, Balasubramanium Saseekaran, MBBS, \\ MD, MRCS, Gayan Bandara, MBBS, MD, MRCS, Rezni Cassim, MBBS, MS and \\ Mandika Wijeyaratne, MBBS, MS, PhD
}

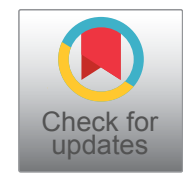

National Hospital of Sri Lanka, Sri Lanka

*Corresponding author: Thilina Gunawardena, MBBS, MD, MRCS, National Hospital of Sri Lanka, 12A, Arawwala Road, Pannipitiya, Sri Lanka, Tel: +94777030315

\begin{abstract}
Pacemaker lead associated venous thrombosis is common. However, due to collateral venous drainage those who are affected rarely become symptomatic. Superior vena cava (SVC) syndrome is a constellation of signs and symptoms that occurs as a result of obstruction to flow in the superior vena cava. In this case report we present a patient who developed this complication following multiple transvenous pacemaker wires. After failed percutaneous venoplasty he was treated successfully with a right internal jugular vein to right atrial appendage bypass.
\end{abstract}

\section{Keywords}

SVC syndrome, Pace maker leads, Lead associated thrombosis

\section{Case Report}

A 57-year-old male patient who was on a permanent pacemaker due to complete heart block presented with slowly progressive swelling of the face and neck. He had severe congestion of the head and neck area when bending down. His first pacing device was implanted in 1992. Due to malfunction, the device was revised twice, in 2005 and 2012. At each revision the existing pacing wires were not extracted.

Physical examination revealed engorged neck veins. Upper limbs were not oedematous. There were no prominent venous collaterals over the anterior chest wall. His basic blood investigations were normal and the recent $2 \mathrm{D}$ echocardiogram showed good biventricular function. Chest X-ray confirmed the presence of multiple pacemaker wires (Figure 1).

According to the clinical findings SVC obstruction was suspected. A CT venogram was done which showed dilated bilateral internal jugular, superior intercostal and azygos veins. The SVC was patent with no definite level of obstruction. However, as the clinical suspicion was high the patient was started on warfarin and oral frusemide. Even though medical therapy for continued more than 6 months, the symptoms progressed to a point where he had to sleep seated due to unbearable congestive symptoms on lying flat.

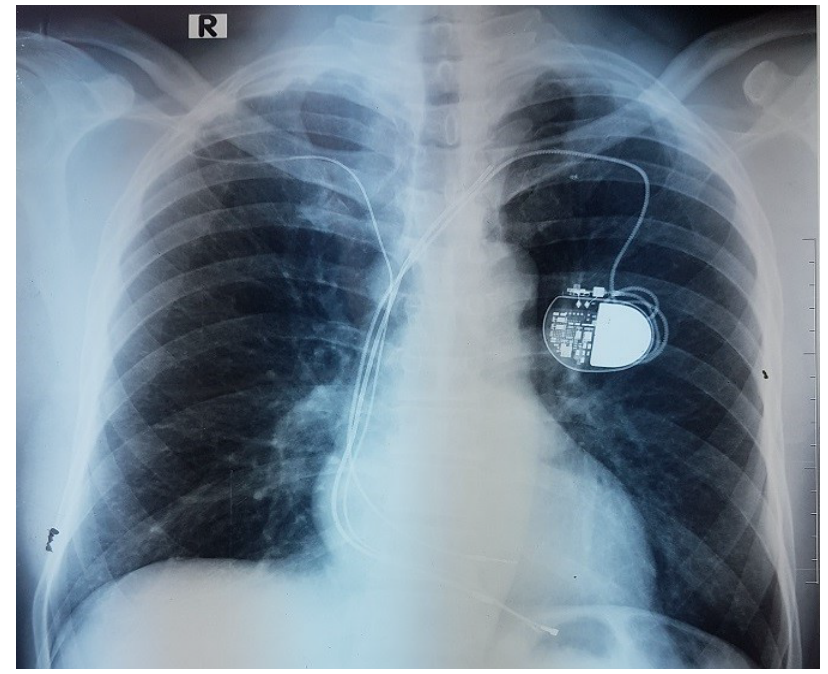

Figure 1: CXR with multiple pacemaker wires.

Citation: Gunawardena T, Saseekaran B, Bandara G, Cassim R, Wijeyaratne M (2020) Pacemaker Lead Associated Superior Vena Cava Syndrome Treated By Veno-Atrial Bypass. Int Arch Cardiovasc Dis 4:030. doi.org/10.23937/2643-3966/1710030

Accepted: June 09, 2020; Published: June 11, 2020

Copyright: (C) 2020 Gunawardena T, et al. This is an open-access article distributed under the terms of the Creative Commons Attribution License, which permits unrestricted use, distribution, and reproduction in any medium, provided the original author and source are credited. 


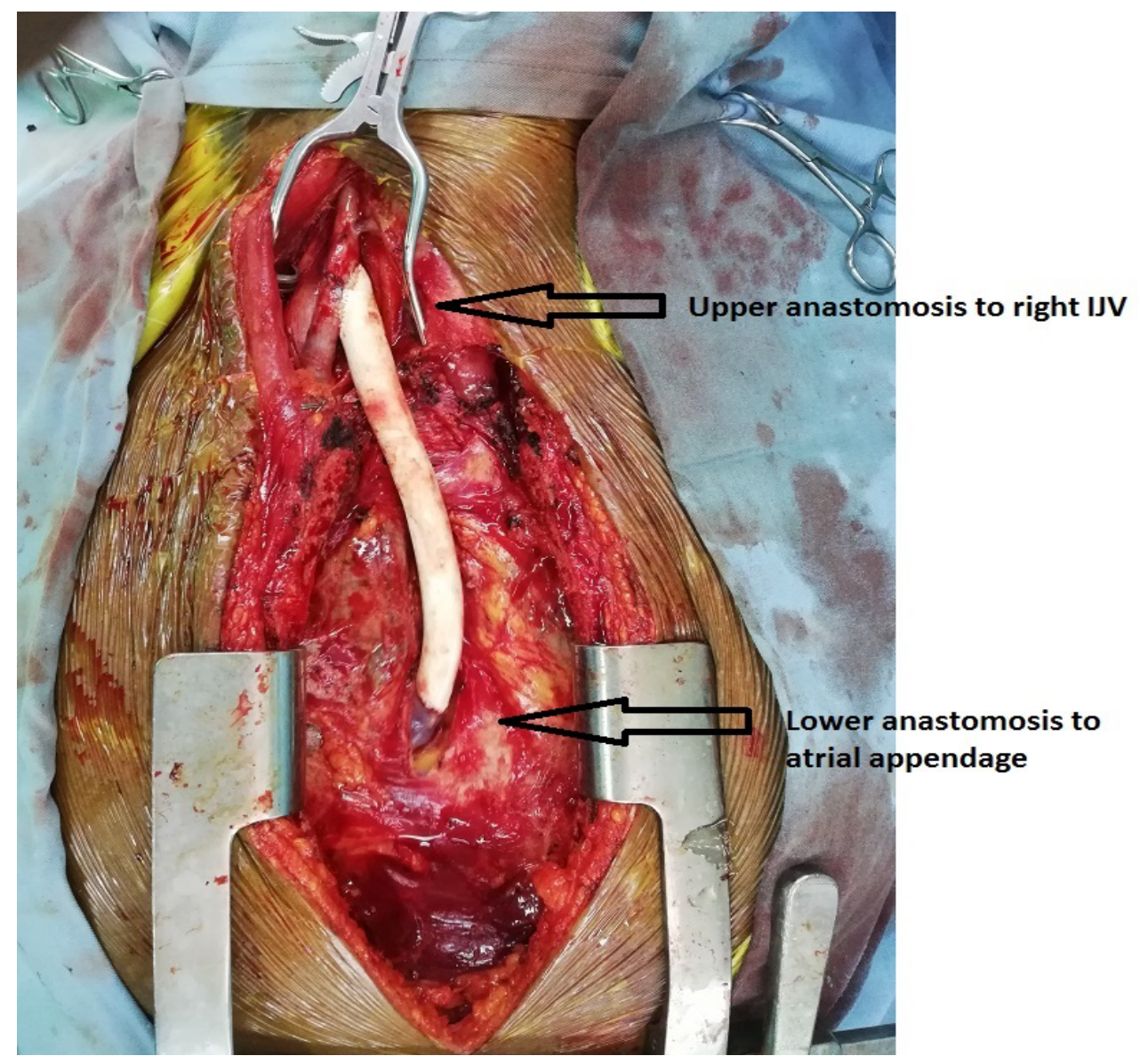

Figure 2: Intra-operative photograph depicting proximal and distal anastomoses.

Then a contrast venogram was arranged which showed $70 \%$ and $50 \%$ narrowing of right and left subclavian veins and $70 \%$ narrowing of the SVC. After multidisciplinary input, a collective decision was taken not to attempt lead extraction because of the prohibitive risk of damaging the central veins. Attempted SVC venoplasty by the interventional radiology team failed due to the presence of multiple pacemaker leads within the SVC. As the symptoms were disabling, the patient was scheduled for a right internal jugular vein to right atrial appendage bypass. The surgery was done under general anesthesia. The right IJV was dissected and the proximal anastomosis was done end to side fashion using a 12 $\mathrm{mm}$ Gore-Tex stretch ${ }^{\circledR}$ vascular graft. Through a midline sternotomy a portion of the right atrial appendage was isolated using a Statinsky clamp. The distal anastomosis was done to this segment (Figure 2). No cardiopulmonary bypass was required. Post-operatively the patient was started on anticoagulation with warfarin and enoxaparin, and enoxaparin was omitted when the target INR of 2-3 was reached. The patient made an uneventful recovery with marked symptom relief following the surgery. He was discharged on warfarin with clinic follow-up. At 6 months from surgery, the graft is patent and the patient remains symptom free.

\section{Discussion}

Pacemaker lead associated venous thrombosis is common. It has an incidence of $30-64 \%$ and the affected majority are asymptomatic [1,2]. However pacemaker leads causing full blown superior vena cava (SVC) syndrome is rare. It occurs in $<0.1 \%$ [3].

The first recorded case of SVC syndrome was by William Hunter in 1757 [4]. The syndrome encompasses a collection of signs and symptoms due to obstruction of blood flow through the SVC [5]. Intrathoracic malignan$\mathrm{cy}$ is the leading cause for this syndrome and accounts for $>90 \%$ of all cases. Historically thoracic aortic aneurysms and mediastinal fibrosis following infection were the commonest benign etiologies. At present iatrogenic SVC syndrome has assumed a more prominent role due to increasing use of intravascular devices such as central venous lines, cardiac pacemakers and implantable cardiac defibrillators [4].

Endothelial injury due to mechanical trauma induced by pacemaker wires triggers the thrombotic process within the vein. Fibrin deposits on the leads and an inflammatory reaction within the vein wall itself contributes to the thrombosis. The end result is total occlusion or fibrotic stenosis of the SVC [6]. The disease process is fairly slow so it allows adequate time for collaterals to develop. Patients become symptomatic when adequate collateralization does not occur [4].

Risk factors for SVC thrombosis in a patient who has 
undergone transvenous pacing are thrombophilic conditions, lead infection, use of hormonal contraceptives, use of a temporary pacing wire before implantation of the permanent device and the presence of multiple active or non-functional pacemaker leads [7]. Our patient had multiple leads within the SVC due to repeated device revisions.

Symptomatic patients with SVC syndrome present with swelling of the face, neck and upper limbs, shortness of breath and orthopnea. Headache, dysphagia, dizziness are other infrequently reported symptoms. The symptoms are the result of impaired venous drainage of head and neck region and upper limbs due to the obstruction in the SVC [4]. Symptoms typically become worse when bending over and lying down. This was noted in our patient as well.

When the diagnosis of SVC syndrome is clinically suspected it should be confirmed by imaging to delineate the location, cause and the extent of the blockage. Chest $X$-ray is not specific and may show a mediastinal mass in those with obstruction due to tumor or aneurysm [4]. CT venography is the first choice of imaging at present and is mandatory to exclude or characterize extrinsic compression of the SVC. Contrast venography is considered the gold standard of imaging but it is invasive. When there is a discordance between clinical findings and CT images as in our case, contrast venography has a place [7]. Magnetic resonance venography is also an imaging option provided the pacemaker device is MRI compatible.

Symptomatic patients with pacemaker lead associated SVC syndrome require treatment and the options are medical management, percutaneous venoplasty or surgery [8]. Medical management includes anticoagulation to prevent clot propagation, diuretics for relief of peripheral oedema, steroids for cerebral/laryngeal oedema and head end elevation of the bed [4]. Those with persistent symptoms despite conservative therapy will need invasive treatment.

The place for routine extraction of non-functional transvenous pacemaker leads in the absence of infection is controversial. The procedure has an associated mortality of $0.4-0.6 \%$ and a morbidity of $1-2 \%$ [9]. Lead associated SVC syndrome is considered an indication for extraction of non-functional, superfluous pacemaker leads $[9,10]$. Removal of the leads per se will not restore the venous patency, so it is unlikely to result in adequate symptom relief [2]. However the channel created in the process of extraction may provide a valuable pathway for a new lead implantation in those with a nonfunctional device and limited access [2,9]. When the gap between the lead implantation and the attempted extraction is prolonged, the lead tends to be well adherent to the vein wall by scar tissue, and pulling the lead out risks damage to the vein wall $[2,11]$. Considering the fact that some of the leads in our patient was im- planted $>2$ decades back and as the current device was functioning well, we decided it was unwise to attempt removal.

Percutaneous intervention with balloon venoplasty with or without stenting is the preferred first line intervention for SVC syndrome at present [3,5]. According to Sfyroeras, et al. $80 \%$ of those who were treated percutaneously required a stent [5]. Thrombolysis can be used as an adjunct before venoplasty in selected cases. Percutaneous interventions in the presence of pacemaker leads can be technically challenging. There is a risk of lead damage and device malfunction which may be life threatening. Retrieval of leads if they get infected becomes difficult when a stent is deployed over them. Covering the procedure with temporary pacing wires, extracting and replacing the leads after the venoplasty and stenting are done are steps that can be taken to circumvent these problems. Patients are usually put on long term anticoagulation therapy following percutaneous treatment [7].

Surgery in the form of bypass grafting has a secondary role at present. It is considered when percutaneous venoplasty fails or when there are complex obstructive lesions not amenable for venoplasty [5]. Native vein grafts, vein allografts, aortic allografts, pericardial tubes and prosthetic grafts have been used as conduits. The long term patencies of native vein grafts are superior to prosthetic grafts. For technical success of bypass grafting size of the conduit should match the inflow vein and the conduit should be of optimum length to avoid redundancy [4]. Patients will need long term anticoagulation after open surgery in order to maintain patency in a low flow bypass.

Short and medium term patency rates after open and after endovascular treatment for SVC syndrome are comparable, but long term patency is better after open surgery. Avoiding a sternotomy is the major advantage of percutaneous intervention over open surgery [5].

\section{Conclusions}

Pacemaker lead associated SVC syndrome is rare. Those with debilitating symptoms despite conservative management will need intervention. Percutaneous balloon venoplasty with or without stenting is the first line approach and surgery assumes a secondary role. Patients will need long term anticoagulation therapy after either form of intervention.

\section{Funding Sources}

None.

\section{Conflicts of Interest}

None.

\section{Authors Contribution}

Thilina Gunawardena designed the manuscript. Thi- 
lina Gunawardena, Balasubramanium Saseekaran and Gayan Bandara wrote the manuscript. Rezni Cassim and Mandika Wijeyaratne supervised the project.

\section{References}

1. Lindsay HS, Chennells PM, Perrins EJ (1994) Successful treatment by balloon venoplasty and stent insertion of obstruction of the superior vena cava by an endocardial pacemaker lead. Br Heart J 71: 363-365.

2. Rozmus G, Daubert JP, Huang DT, Rosero S, Hall B, et al. (2005) Venous thrombosis and stenosis after implantation of pacemakers and defibrillators. J Interv Card Electrophysiol 13: 9-19.

3. Aryana A, Sobota KD, Esterbrooks DJ, Gelbman AI (2007) Superior vena cava syndrome induced by endocardial defibrillator and pacemaker leads. Am J Cardiol 99: 17651767.

4. Mohamed A, El M, Amin M (2009) Superior vena cava syndrome: A systematic review. The Journal of the Egyptian Medical Association.

5. Sfyroeras GS, Antonopoulos CN, Mantas G, Moulakakis KG, Kakisis JD, et al. (2017) A Review of open and en- dovascular treatment of superior vena cava syndrome of benign aetiology. Eur J Vasc Endovasc Surg 53: 238-254.

6. Senthilvel E, Papadakis A, Jain V, Bruner J (2009) Pacemaker induced superior vena cava syndrome: A case report. Cases J 2: 6463.

7. Klop B, Scheffer MG, McFadden E, Bracke F, van Gelder B (2011) Treatment of pacemaker-induced superior vena cava syndrome by balloon angioplasty and stenting. Neth Heart J 19: 41-46.

8. Slonim SM, Semba CP, Sze DY, Dake MD (2000) Placement of SVC stents over pacemaker wires for the treatment of SVC syndrome. J Vasc Interv Radiol 11: 215-219.

9. Henrikson CA (2010) Think before you pull-not every lead has to come out. Circ Arrhythm Electrophysiol 3: 409-412.

10. Bongiorni MG, Burri H, Deharo JC, Starck C, Kennergren C, et al. (2018) 2018 EHRA expert consensus statement on lead extraction: Recommendations on definitions, endpoints, research trial design, and data collection requirements for clinical scientific studies and registries: Endorsed by APHRS/HRS/LAHRS. Europace 20: 1217.

11. Bracke $F(2008)$ Complications and lead extraction in cardiac pacing and defibrillation. Neth Heart J 16: S28-S31. 